\title{
Prevalencia de Porcentaje de Grasa Corporal, Obesidad Abdominal y Estado Nutricial en una Escuela Primaria de Mexicali Baja California México
}

\author{
Prevalence of Body Fat, Abdominal Obesity and Nutritional State in the \\ Students of an Elementary School from Mexicali Baja California Mexico
}

\author{
Javier Arturo Hall López*; Paulina Yesica Ochoa Martínez*; Juan Carlos Borbón Román* \& Luis Roberto Monreal Ortíz**
}

HALL, L. J. A.; OCHOA, M. P. Y.; BORBÓN, R. J. C. \& MONREAL, O. L. R. Prevalencia de porcentaje de grasa corporal, obesidad abdominal y estado nutricio en una escuela primaria de Mexicali Baja California México. Int. J. Morphol., 31(4):1269-1275, 2013.

RESUMEN: El objetivo de este estudio fue estimar la prevalencia del porcentaje de grasa corporal, obesidad abdominal y estado nutricional en los estudiantes de la Escuela Primaria Profesor Salvador Jiménez Gómez de Mexicali Baja California, México. Fueron evaluados 621 estudiantes, con un rango de edad de 6 a 13 años. A cada niño se le tomaron, medidas antropométricas de peso, talla, circunferencia de cintura, pliegue de tríceps y de pantorrilla; se utilizó el programa Epi Info Versión 3.3.2 para calcular el índice de masa corporal (IMC), estableciendo como obesidad un valor mayor o igual al percentil 95 y el sobrepeso un valor mayor o igual al percentil 85 y menor al 95 de acuerdo con la edad y sexo, tomando en cuenta la medición de circunferencia de cintura se determinó obesidad abdominal mayor o igual al percentil 90 de acuerdo con la edad y sexo; el porcentaje de grasa corporal de determino por la ecuación de slaughter. Las variables con prevalencias más altas resultaron las del estado nutricional, con donde el 19,1\% de la población estudiada presentó sobrepeso y $25,1 \%$ obesidad. La prevalencia combinada de sobrepeso y obesidad en niños fue de $51,3 \%$ mientras que en niñas 37,4\%. Los resultados encontrados en estos niños muestran claramente altas prevalencias de sobrepeso y obesidad. Inclusive más altas al promedio de los niños mexicanos. Concluyendo que en esta escuela es necesario tomar medidas, como poner en marcha programas de actividad física y nutrición orientados a frenar el problema del sobrepeso y obesidad.

PALABRAS CLAVE: Grasa Corporal; Obesidad; Estado nutricio; Estudiantes primarios.

\section{INTRODUCCIÓN}

La obesidad es un problema de salud creciente que afecta cada vez a más niños en el mundo, sin importar sexo, grupo étnico o nivel socioeconómico (Ebbeling et al., 2012). Como principal referente tenemos el caso de Estados Unidos, país que reporta la más alta prevalencia de obesidad infantil a nivel mundial $(17,1 \%)$, donde en los niños de 6 a 11 años de edad, la prevalencia de obesidad se ha incrementado progresivamente de $4,1 \%$ a $10,7 \%$, de 1963 a 1994, con similar incremento en jóvenes de 12 a 17 años (4,6\% a 10,7\%) (Ogden et al., 2006; Troiano \& Flegal, 1988). Cabe resaltar que los adolescentes mexicanos que viven en Estados Unidos son los que presentan las tasas de sobrepeso y obesidad más altas en ese país, los norteamericanos caucásicos tienen una prevalencia del $26 \%$, mientras los México-americanos presentan una cifra de $44 \%$ (Ogden et al., 2002).
La prevalencia de obesidad en niños mexicanos menores de 5 años aumentó de 4,2\% a 5,3\% de 1988 a 1995, así mismo los resultados de la última Encuesta Nacional de Salud y Nutrición (ENSANUT) en México (Olaiz et al., 2006), reportan que la obesidad en escolares de 5 a 11 años de edad, aumentó de $5,3 \%$ a $9,4 \%$ en niños y de $5,9 \%$ a 8,7\% en niñas de 1999 al 2006 mostrando con ello una prevalencia combinada de sobrepeso y obesidad en aumento, pasando de $18,6 \%$ a 26,3\% (Rivera et al., 2002; Rivera et al., 2006). La región norte de México es considerada como la más alta en prevalencia de obesidad y sobrepeso de niños de edad escolar con un 35,1\% (Hernández et al., 2001). La ENSANUT, reportó un incremento en la prevalencia de obesidad y sobrepeso en mujeres de 12 a 19 años pasando de $6,9 \%$ a $9,2 \%$ y de $21,6 \%$ a $23,3 \%$ respectivamente en el periodo de 1999 a 2006, mientras que en el sexo masculino

\footnotetext{
* Escuela de Deportes Universidad Autónoma de Baja California (UABC), México.

** Escuela Superior de Educación Física, Universidad Autónoma de Sinaloa (UAS), México.
} 
de la misma edad, no se reportaron cifras a nivel nacional del aumento a través del tiempo, sin embargo en los datos de la encuesta se observa la prevalencias de obesidad de $10 \%$ y sobrepeso de $21,2 \%$ (Rivera et al., 2006).Un estudio derivado de la Encuesta Nacional de Salud 2000 reporta en jóvenes de 10 a 17 años de edad, una prevalencia de obesidad y sobrepeso de $7,7 \%$ y $17 \%$ en hombres y $6,9 \%$ y $20,6 \%$ en mujeres (del Rio-Navarro et al., 2004).

Diversos estudios del estado nutricional, realizados en niños y jóvenes mexicanos muestran que en diferentes contextos de nuestro país la prevalencia de sobrepeso y obesidad presenta valores variados (muestras y/o poblaciones del Norte, Centro y Sur de México, comunidades fronterizas, urbanas, suburbanas, marginales y México-Americanas) (Tabla I).

En los últimos años México al igual que muchos países, ha experimentado cambios en el estilo de vida a consecuencia de la urbanización. Dichos cambios incluyen la adopción de dietas altas en energía, con altos contenidos de grasa, azúcares refinados, así como una disminución de la actividad física, presentando en mayor medida consecuencias patológicas, psicológicas y sociales relacionadas a la obesidad infantil (Freedman et al., 1999; Hill \& Silver, 1995; Lizana et al., 2011; Carrasco et al., 2011).

El impacto de la obesidad en niños y adolescentes se mide tanto por las consecuencias patológicas que presenta en el momento presente, así como las complicaciones que presentan en etapas posteriores con la edad, esto debido a que un $80 \%$ de los adolescentes con obesidad, se convierten en adultos con obesidad (Kibbe \& Offner, 1999). En los adultos, numerosos estudios epidemiológicos han demostrado la relación directa entre la obesidad y enfermedades como hipertensión, diabetes, dislipidemias, cáncer, cardiopatía isquémica, entre otras no trasmisibles (Bray, 1999).

\section{MATERIAL Y MÉTODO}

Población. El presente estudio incluyó a 621 estudiantes de la Escuela Primaria Profesor Salvador Jiménez Gómez de Mexicali Baja California. México. Con un rango de edad de 6 a 13 años. Los cuales contaron con la autorización de participación voluntaria de sus padres y autoridades educativas de la escuela.

Instrumentos y Procedimiento. A cada niño se le tomaron, las medidas antropométricas de peso, talla, circunferencia de cintura, pliegue de tríceps y pliegue de pantorrilla, para determinar el peso se utilizó una báscula digital marca Tanita con capacidad de $140 \mathrm{Kg}$ y una precisión de $100 \mathrm{~g}$ y un estadiómetro de pared, una cinta metálica inextensible para la obtención de la circunferencia de cintura y un plicómetro Slim Guide para la obtención de los pliegues cutáneos. Se usó el programa Epi Info Versión 3.3.2 que maneja las referencias del CDC 2000 (Centro para el control y la prevención de las enfermedades por sus siglas en ingles) para calcular, el índice de masa corporal [IMC Genero-Edad= peso $(\mathrm{kg}) /$ talla al cuadrado $\left(\mathrm{m} \sum\right)$ ], estableciendo el estado

Tabla I. Estudios de prevalencia de sobrepeso y obesidad infantil en México.

\begin{tabular}{|c|c|c|c|c|}
\hline Lugar & Población & edad & Prevalencia de obesidad & Referencia \\
\hline Tezontepec de Aldama & 400 & $6-12$ & $51 \%$ peso bajo & Castañeda et al., 2002. \\
\hline Hidalgo & & & $18 \%$ sobrepeso y obesidad & \\
\hline Delegación Milpa Alta D.F. & 303 & $11-13$ & $\begin{array}{l}29.4 \% \text { Sobrepeso } \\
7.6 \% \text { obesidad } \\
37 \% \text { sobrepeso y obesidad }\end{array}$ & Hernández et al., 2001. \\
\hline El paso, Texas & 1370 & $8-9$ & $28 \%$ de sobrepeso y & Coleman et., 2004. \\
\hline Mineral del Chico, Pachuca & 264 & $8-18$ & $23.9 \%$ de sobrepeso y & Villanueva \& Moreno, 2004. \\
\hline Hermosillo sonara & 254 & $6-10$ & $22 \%$ de sobrepeso y & Ramírez-López et al., 2005 \\
\hline Chilpancingo Guerrero & 622 & $5-10$ & $\begin{array}{l}28.1 \% \text { de sobrepeso } \\
13.7 \% \text { de obesidad } \\
41.8 \% \text { de sobrepeso y }\end{array}$ & Alves de Moraes et al., 2006. \\
\hline $\begin{array}{l}\text { Río grande Valley, Texas. } \\
\text { Matamoros Tamaulipas }\end{array}$ & 5389 & $15-19$ & $\begin{array}{l}16 \% \text { de sobrepeso } \\
17 \% \text { de obesidad } \\
33 \% \text { Obesidad y } \\
\text { sobrepeso. }\end{array}$ & Pérez et al., 2006. \\
\hline
\end{tabular}


nutricional, como obesidad $\geq$ al percentil 95 , el sobrepeso $\geq$ al percentil $85 \mathrm{y}<$ al 95, el peso normal $\geq$ al percentil $5 \mathrm{y}<$ al 85 y el peso bajo < al percentil 5. Se clasificó con obesidad abdominal quien presentó valores $\geq$ al percentil noventa de acuerdo a su edad y sexo, el porcentaje de grasa corporal se clasifico mediante diversos puntos de corte en catego-

Tabla II. Características generales de los niños de la Escuela Primaria Profesor Salvador Jiménez Gómez, de Mexicali baja California.

\begin{tabular}{lcccc}
\hline Caracterís tica & Media & D. S. & $\begin{array}{l}\text { Rango } \\
\text { Mínimo }\end{array}$ & $\begin{array}{c}\text { Rango } \\
\text { máximo }\end{array}$ \\
\hline Edad (años) & 8.6 & 1.5 & 6 & 12 \\
Peso $(\mathrm{kg})$ & 36.8 & 12.9 & 17 & 76 \\
Estatura $(\mathrm{cm})$ & 134.5 & 12.1 & 106 & 165 \\
IMC $\left(\mathrm{kg} / \mathrm{m}^{2}\right.$ & 19.8 & 4.5 & 12.3 & 34.2 \\
Percentil & 71.5 & 30.4 & 0 & 99.9 \\
Pliegue de tríceps $(\mathrm{mm})$ & 17.2 & 6.7 & 4.0 & 42 \\
Pliegue de pantorrilas $(\mathrm{mm})$ & 16.2 & 6.4 & 4.0 & 35 \\
\% de grasa corporal & 26.2 & 9.7 & 8.4 & 57.6 \\
Circunferen. de cintura $(\mathrm{cm})$ & 68.4 & 12.6 & 40.1 & 103.2 \\
\hline
\end{tabular}

rías de riesgo (Como Muy Bajo, Bajo, Rango Óptimo, Moderadamente Alto, Alto Muy Alto), validado por Slaughter et al. (1988). Con los resultados obtenidos se elaboro una base de datos en el programa SPSS Versión 15.0 para su procesamiento estadístico.

\section{RESULTADOS}

Tabla III. Características generales de las niñas de la Escuela Primaria Profesor Salvador Jiménez Gómez, de Mexicali baja California..

\begin{tabular}{lcccc}
\hline Caracterís tica & Media & D. S. & $\begin{array}{l}\text { Rango } \\
\text { Mínimo }\end{array}$ & $\begin{array}{c}\text { Rango } \\
\text { máximo }\end{array}$ \\
\hline Edad (años) & 8.6 & 1.7 & 6 & 12 \\
Peso $(\mathrm{kg})$ & 34.3 & 12.8 & 15 & 78 \\
Estatura $(\mathrm{cm})$ & 133.4 & 13.5 & 102 & 164 \\
IMC $\left(\mathrm{kg} / \mathrm{m}^{2}\right.$ & 18.6 & 4.3 & 11.9 & 31.9 \\
Percentil & 59.8 & 34.6 & 0 & 99.9 \\
Pliegue de tríceps (mm) & 15.7 & 6.5 & 5 & 39 \\
Pliegue de pantorrillas & 14.6 & 6.5 & 3 & 41 \\
\% de grasa corporal & 23.6 & 7.4 & 10.6 & 53.9 \\
Circunferen. de cintura & 65.5 & 11.7 & 38.2 & 105.5 \\
\hline
\end{tabular}

Tabla IV. Distribución porcentual de la población de acuerdo a edad y sexo, según el índice de masa corporal $\left(\mathrm{kg} / \mathrm{m}^{2}\right)$ puntos de corte de la CDC 2000 (N=622) de los niños de la Escuela Primaria Profesor Salvador Jiménez Gómez, de Mexicali baja California.

\begin{tabular}{|c|c|c|c|c|}
\hline & $\begin{array}{c}\text { Peso bajo percentil } \\
(<5)\end{array}$ & $\begin{array}{l}\text { Peso normal percentil } \\
\qquad(\geq 5 \mathrm{y}<85)\end{array}$ & $\begin{array}{l}\text { Sobrepeso percentil } \\
\quad(\geq 85 \mathrm{y}<95)\end{array}$ & $\begin{array}{c}\text { Obesidad } \\
\text { percentil }(>95)\end{array}$ \\
\hline Niños de 6 años & $\%$ & $\%$ & $\%$ & $\%$ \\
\hline Hombres & 3.1 & 53.1 & 21.9 & 21.9 \\
\hline Mujeres & 14.6 & 56.1 & 12.2 & 17.1 \\
\hline Total & 9.6 & 54.8 & 16.4 & 19.2 \\
\hline \multicolumn{5}{|l|}{ Niños de 7 años } \\
\hline Hombres & 2 & 51 & 16.3 & 30.6 \\
\hline Mujeres & 8.2 & 72.1 & 8.2 & 11.5 \\
\hline Total & 5.5 & 62.7 & 11.8 & 20 \\
\hline \multicolumn{5}{|l|}{ Niños de 8 años } \\
\hline Hombres & 3.4 & 44.1 & 27.1 & 25.4 \\
\hline Mujeres & 4.1 & 32.7 & 26.5 & 36.7 \\
\hline Total & 3.7 & 38.9 & 26.9 & 30.6 \\
\hline \multicolumn{5}{|l|}{ Niños de 9 años } \\
\hline Hombres & 4.6 & 49.2 & 20 & 26.2 \\
\hline Mujeres & 12.5 & 52.5 & 20.0 & 15 \\
\hline Total & 7.6 & 50.5 & 20 & 21.9 \\
\hline \multicolumn{5}{|l|}{ Niños de 10 años } \\
\hline Hombres & 3.9 & 56.9 & 11.8 & 27.5 \\
\hline Mujeres & 15.2 & 59.1 & 10.6 & 15.2 \\
\hline Total & 10.3 & 58.1 & 11.1 & 20.5 \\
\hline \multicolumn{5}{|c|}{ Niños de 11 años y más } \\
\hline Hombres & 2 & 20 & 32 & 46 \\
\hline Mujeres & 5.1 & 40.7 & 25.4 & 28.8 \\
\hline Total & 3.7 & 31.2 & 28.4 & 36.7 \\
\hline Hombres to tal & 3.3 & 45.4 & 21.6 & 29.7 \\
\hline
\end{tabular}


Tabla V. Distribución del porcentaje de grasa corporal en niños de ambos sexos de la Escuela Primaria Profesor Salvador Jiménez Gómez, de Mexicali baja California.

\begin{tabular}{lccccc}
\hline $\mathbf{N}=\mathbf{3 0 8}$ & Bajo & Rango & Moderadamente & Alto & Muy alto \\
\hline Hombres n= 156 & & & & & \\
Mujeres n=152 & & & & & \\
\hline Niños de 9 años & 1.8 & 36.4 & 10.9 & 25.5 & 25.5 \\
Hombres & 0 & 54.1 & 21.6 & 16.2 & 8.1 \\
Mujeres & 1.1 & 43.5 & 15.2 & 21.7 & 18.5 \\
\hline Total & & & & & \\
\hline Niños de 9 años & 2.0 & 28.0 & 18.0 & 24.0 & 28.0 \\
Hombres & 4.5 & 54.5 & 19.7 & 13.6 & 7.6 \\
Mujeres & 3.4 & 43.1 & 19 & 18.1 & 16.4 \\
\hline Total & & & & & \\
\hline Niños de 9 años & 2.1 & 23.4 & 19.1 & 10.6 & 44.7 \\
Hombres & 3.8 & 62.3 & 13.2 & 20.8 & 0.0 \\
Mujeres & 3 & 44 & 16 & 16 & 21 \\
\hline Total & 2.0 & 29.6 & 15.8 & 20.4 & 32.2 \\
\hline Hombres total & 3.2 & 57.1 & 17.9 & 16.7 & 5.1 \\
Mujeres total & 2.6 & 43.5 & 16.9 & 18.5 & 18.5 \\
\hline Total & & & & & \\
\hline
\end{tabular}

Tabla VI. Distribución percentilar de la circunferencia de cintura de los alumnos de la Escuela Primaria Profesor Salvador Jiménez Gómez, de Mexicali baja California.

\begin{tabular}{lllllllll}
\hline $\begin{array}{l}\text { Edad y Sexo } \\
\text { Masculino n=306 }\end{array}$ & $\mathbf{p 5}$ & $\mathbf{p 1 0}$ & $\mathbf{p 2 5}$ & $\mathbf{p 5 0}$ & $\mathbf{p 7 5}$ & $\mathbf{p 8 5}$ & $\mathbf{p 9 0}$ & $\mathbf{p 9 5}$ \\
\hline 6 & 50 & 50.8 & 53 & 55 & 59 & 65.4 & 71.4 & 73 \\
7 & 42.5 & 51 & 54.5 & 59 & 67 & 73 & 83 & 88 \\
8 & 51 & 52 & 56 & 64.5 & 70 & 71 & 74.7 & 82 \\
9 & 54.1 & 57 & 60.75 & 67 & 79 & 82 & 85 & 91.8 \\
10 & 55.7 & 57.4 & 61.5 & 68 & 78.5 & 84.8 & 87.2 & 95 \\
11 y más & 55.8 & 59.9 & 66.75 & 81.5 & 91.5 & 93 & 97.1 & 99 \\
Total & 52 & 53 & 58 & 65 & 76 & 82 & 86 & 93 \\
\hline Femenino n=316 & $\mathbf{p 5}$ & $\mathbf{p 1 0}$ & $\mathbf{p 2 5}$ & $\mathbf{p 5 0}$ & $\mathbf{p 7 5}$ & $\mathbf{p 8 5}$ & $\mathbf{p 9 0}$ & $\mathbf{p 9 5}$ \\
6 & 46 & 47.8 & 51 & 53.5 & 60.25 & 66 & 66.4 & 70 \\
7 & 49 & 50 & 52 & 56 & 60 & 65 & 72 & 77 \\
8 & 51.8 & 55 & 60 & 66 & 74.5 & 79.3 & 80.2 & 85.2 \\
9 & 50.5 & 53 & 56.25 & 66 & 72.75 & 77 & 79 & 85.75 \\
10 & 49 & 54 & 58 & 65 & 76 & 85 & 87 & 91 \\
11 y más & 54.4 & 55.4 & 61.5 & 70 & 83 & 86 & 90 & 91.9 \\
\hline Total & 50 & 52 & 56 & 63 & 73 & 79.3 & 84.2 & 87 \\
\hline
\end{tabular}

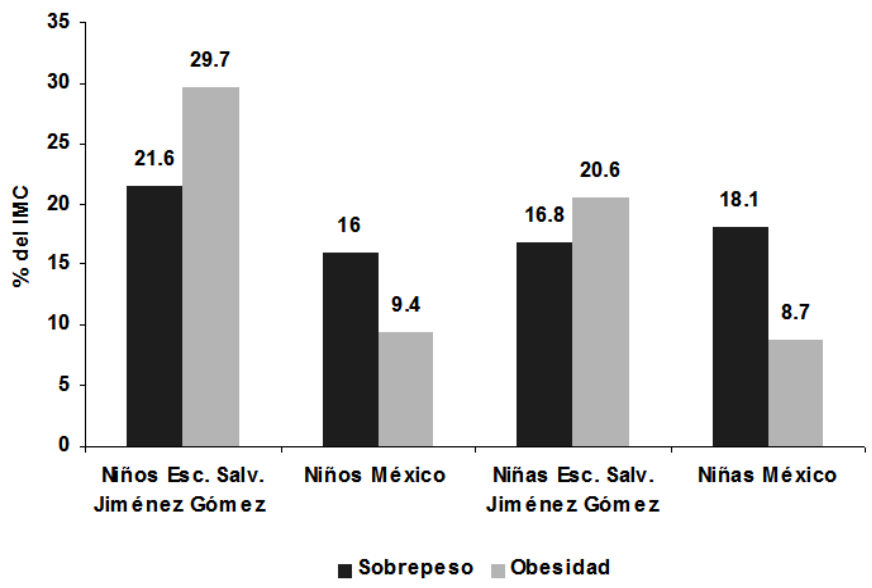

Fig. 1. Comparativo por sexo de la prevalencia nacional de sobrepeso y obesidad en población de 5 a 11 años de edad de la Encuesta Nacional de Salud y Nutrición 2006 (ENSANUT 2006), México, y los alumnos de la Escuela Primaria Profesor Salvador Jiménez Gómez, de Mexicali Baja California. 


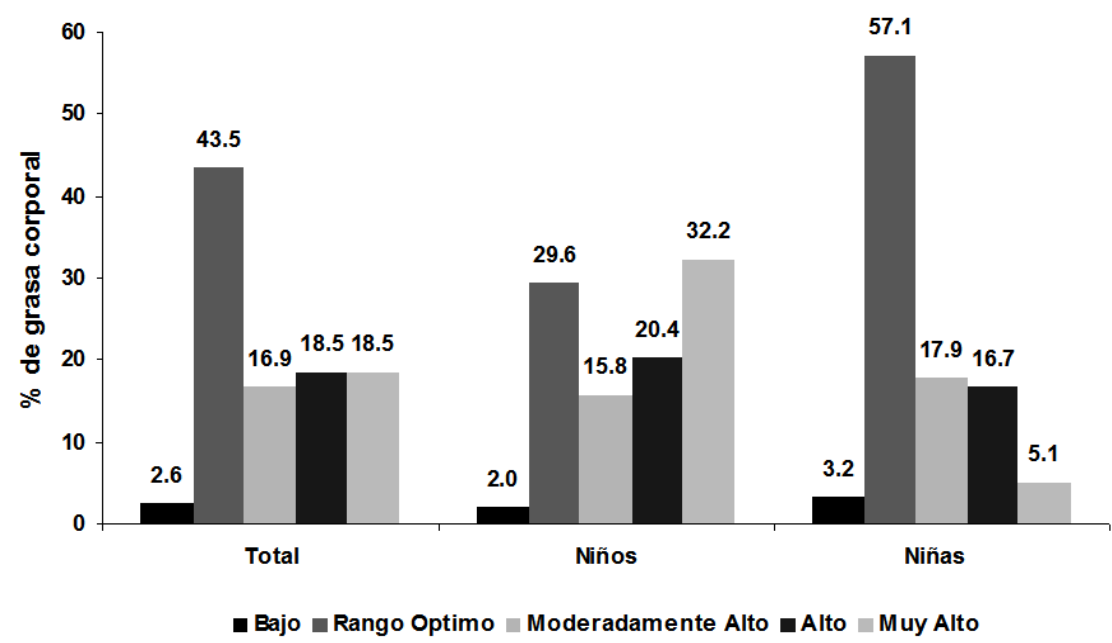

Fig. 2. Distribución porcentual de grasa corporal en los niños 9 a 12 años de edad de la Escuela primaria Profesor Salvador Jiménez Gómez, de Mexicali Baja California.

Fig. 3. Distribución del porcentaje de obesidad abdominal en los niños de la Escuela primaria Profesor Salvador Jiménez Gómez, de Mexicali Baja California.
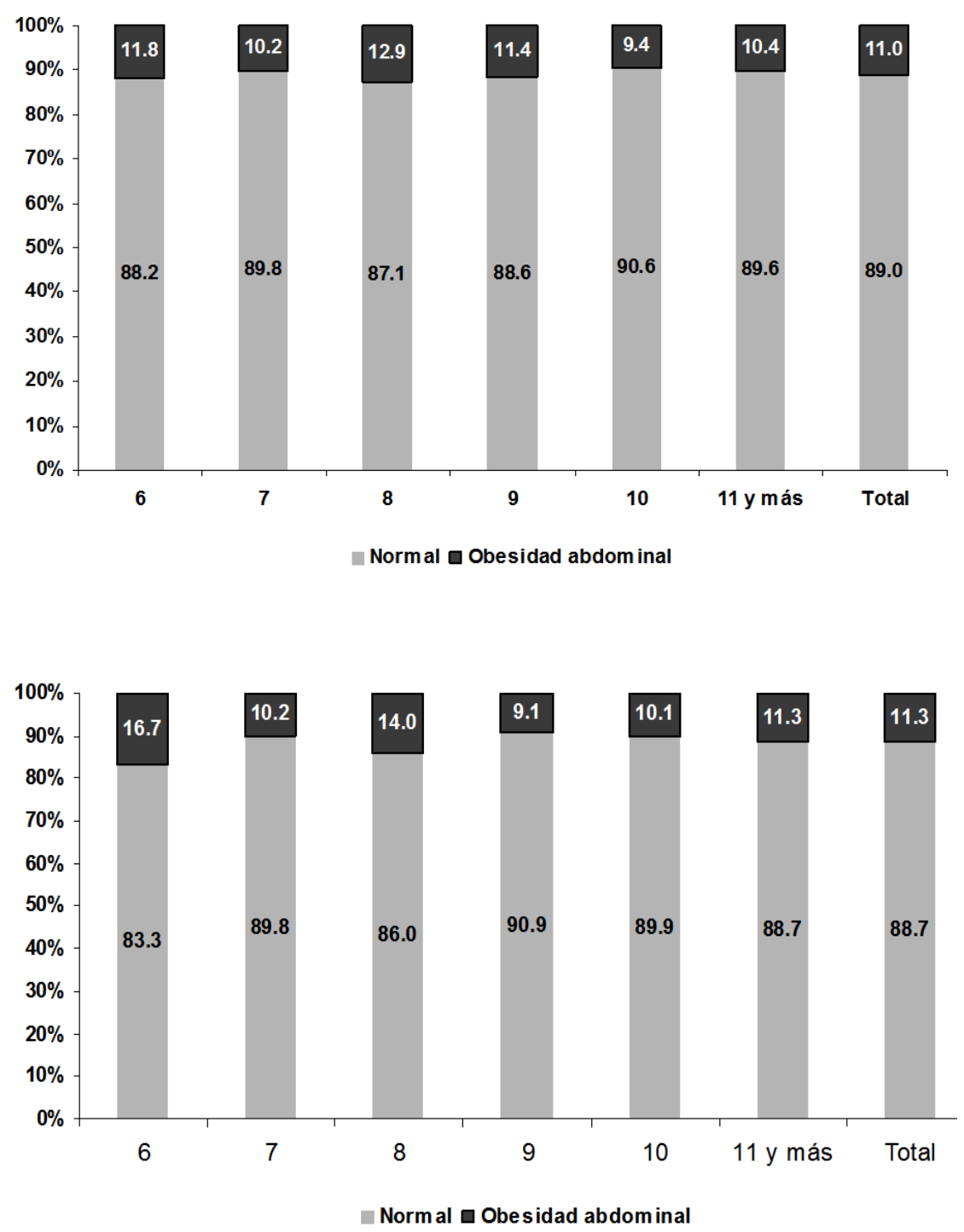

Fig. 4. Distribución del porcentaje de obesidad abdominal en las niñas de la Escuela Primaria Profesor Salvador Jiménez Gómez, de Mexicali Baja California. 


\section{DISCUSIÓN}

Se advierte que clasificaciones de obesidad abdominal resultaron con mayor prevalencia en niñas que en los niños, por lo contrario al determinar el porcentaje de grasa corporal las clasificaciones combinadas de moderadamente alto, alto y muy alto resultaron mayores en los niños que en las niñas, de igual manera el estado nutricional donde la prevalencia de sobrepeso es mayor en niños que en las niñas, sin embargo, en lo referente a la obesidad, los resultados se invierten y las niñas presentan menor prevalencia que los niños, no obstante en términos generales la población estudiada muestra una prevalencia de sobrepeso y obesidad más alta respecto a la media nacional según los reportes de la más reciente ENSANUT realizada en México (Olaiz et al.), con el considerando de que en este último estudio se utilizaron los criterios para estimar el estado nutricio propuestos por el International Obesity Task Force (IOTF).

La primera estrategia para contener el problema del sobrepeso y obesidad es el diagnóstico. Podemos señalar que los alumnos de la Escuela Primaria Profesor Salvador Jiménez Gómez de Mexicali Baja California, presentan problemas de sobrepeso y obesidad, sin embargo la magnitud de este problema no radica solamente en su alta prevalencia si no, en su relación con el riesgo a padecer enfermedades metabólicas y cardiovasculares prematuras que puede repercutir en su calidad de vida (Pearce et al., 2002) siendo el medio escolar ideal por su amplia cobertura el que se reco- mienda a nivel mundial para la promoción de actitudes positivas para desarrollar en los niños hábitos saludables de alimentación y actividad física (Sallis \& Glanz, 2009).

En México se han aplicado programas exitosos en el medio escolar que han demostrado resultados efectivos al utilizar estrategias para prevenir factores de riesgo cardiovascular mediante componentes de hábitos saludables y actividad físicas (Perichart-Perera et al., 2008; ColinRamirez et al., 2010), esto a futuro coadyudará a generar en los niños un estilo de vida saludable fomentando que se adopte y que conserve en edades posterioresde la vida y que a futuro se reduzcan los altos costos en tratamientos relacionados con la morbilidad y mortalidad de enfermedades relacionadas con la obesidad (Story et al., 2009).

\section{CONCLUSIONES}

En la población estudiada es urgente aplicar medidas que conduzcan a la disminución del sobrepeso y obesidad, siendo necesario que los profesionales de la salud diseñen y operen alternativas efectivas para la prevención y tratamiento integral de la obesidad infantil y minimizar las posibles consecuencias derivadas de las misma, considerando el contexto específico de la educación básica y sus características particulares, así como los entornos socioeconómicos y culturales de las personas que acuden a la educación pública en este nivel.

HALL, L. J. A.; OCHOA, M. P. Y.; BORBÓN, R. J. C. \& MONREAL, O. L. R. Prevalence of body fat, abdominal obesity and nutritional state in the students of an elementary school in Mexicali Baja California, México. Int. J. Morphol., 31(4):1269-1275, 2013.

SUMMARY: The aim of this work was to estimate the prevalence of body fat, abdominal obesity and nutritional state in the students of the elementary school Salvador Jimenez Gomez in Mexicali Baja California. Mexico. Six hundred twenty-one children of 6 to 13 years old were studied, to determine the body fat, abdominal obesity and nutritional state, anthropometric measurements were taken and medial calf skinfolds, waist circumference, weight and height, body fat was determined according to the slaughter equation, abdominal obesity was determined $\geq 90$ percentile according whit gender and age and Epi Info version 3.3.2 software was used to classify the overweight $(\geq 85$ th percentile and $<95$ th percentile BMI for age and gender) and obesity ( $\geq 95$ th percentile BMI for age and gender) and through the body mass index (BMI). In our results, 19.1\% of the students had overweight and $25.1 \%$ obesity. Combined overweight and obesity prevalence in boys was $51.3 \%$ while in girls was $37.4 \%$. These results clearly show high prevalence of overweight and obesity in these children, in addition the children of this study were more overweight and obese than Mexican children average. It concludes that efforts should be made in this school to develop physical activity and nutrition programs to help stem rates of overweight and obesity.

KEY WORDS: Body fat; Overweight; Nutritional state; Elementary school students.

\section{REFERENCIAS BIBLIOGRÁFICAS}

Alves, S.; Beltran, J.; Mondini, L. \& Martins, I. Prevalência de sobrepeso e obesidade e fatores asociados em escolares de área urbana de Chilpancingo, Guerrero, México, 2004. Cad. Saúde Pública, 22(6):1289-301, 2006.

Bray, G. A. Medical consequences of obesity. J. Clin. Endocrinol. Metab., 89(6):2583-9, 2004
Carrasco,V.; Martínez, C.; Silva, H.; Collipal, E.; Campos, C. \& Silva, T. Prevalence of obesity in a sample of schoolchildren from municipalized schools in the IX Region of Chile 2008-2009. Int. J. Morphol., 29(3):830-4, 2011.

Castañeda, R.; Molina, N. \& Hernández, J. Estado nutricional de escolares en una población del estado de Hidalgo, México. Rev. Endocrinol. Nutr., 10(4):201-5, 2002. 
HALL, L. J. A.; OCHOA, M. P. Y.; BORBÓN, R. J. C. \& MONREAL, O. L. R. Prevalencia de porcentaje de grasa corporal, obesidad abdominal y estado nutricio en una escuela primaria de Mexicali Baja California México. Int. J. Morphol., 31(4):1269-1275, 2013.

Coleman, K. J.; Heath, E. M. \& Alcalá, I. S. Overweight and aerobic fitness in children in the United States/Mexico border region. Rev. Panam. Salud Publica, 15(4):262-71, 2004.

Colin-Ramírez, E.; Castillo-Martínez, L.; Orea-Tejeda, A.; VergaraCastañeda, A.; Keirns-Davis, C. \& Villa-Romero, A. Outcomes of a school-based intervention (RESCATE) to improve physical activity patterns in Mexican children aged 8-10 years. Health Educ. Res., 25(6):1042-9, 2010.

del Rio-Navarro, B. E.; Velázquez-Monroy, O.; Sánchez-Castillo, C. P.; Lara-Esqueda, A.; Berber A.; Fanghänel, G.; Violante, R.; TapiaConyer, R.; James, W. P. \& Encuesta Nacional de Salud 2000 Working Group, National Health Survey 2000. The ENSA 2000 Working Group. The high prevalence of overweight and obesity in Mexican children. Obes. Res., 12(2):215-23, 2004.

Ebbeling, C. B.; Pawlak, D. B. \& Ludwig, D. S. Childhood obesity: public-health crisis, common sense cure. Lancet, 360(9331):47382, 2002.

Freedman, D. S.; Dietz, W. H.; Srinivasan, S. R. \& Berenson, G. S. The relation of overweight to cardiovascular risk factors among children and adolescents: The Bogalusa Heart Study. Pediatrics, 103(6 Pt. 1):1175-82, 1999

Hernández, B.; Dommarco, J.; Shamah, T.; Cuevas, L.; Ramírez, I. \& Camacho. M. Encuesta Nacional de Nutrición 1999 Estado nutricio de niños y mujeres en México. Cuernavaca, México, Instituto Nacional de Salud Pública, 2001. pp.69-101.

Hill, A. J. \& Silver, E. K. Fat, friendless and unhealthy: 9-year old children's perception of body shape stereotypes. Int. J. Obes. Relat. Metab. Disord., 19(6):423-30, 1995.

Kibbe, D. \& Offner, R. Childhood Obesity - Advancing Effective Prevention and Treatment: An Overview for Health Professionals. Prepared for the National Institute for Health Care Management Foundation Forum, 2003. Disponible en: http://www.nihcm.org/ ChildObesityOverview.pdf

Lizana, A. J. P.; Almagia, F. A. A.; Simpson, L. M. C. Binvignat, G. O. Ivanovic, M. D. \& Berral De La Rosa, F. J. Approximation to the Secular Tendency of the Nutritional State and Body Composition of High School Students, V Region, Chile: 1985-2010. Int. J. Morphol., 29(2):473-8, 2011.

Pearce, M. S.; Basterfield, L.; Mann, K. D.; Parkinson, K. N.; Adamson, A. J.; Reilly, J. J. \& Gateshead Millennium Study Core Team. Early predictors of objectively measured physical activity and sedentary behaviour in 8-10 year old children: The Gateshead Millennium Study. PLoS One, 7(6):e37975, 2012.

Pérez, A.; Reininger, B. M.; Aguirre Flores, M. I.; Sanderson, M. \& Roberts, R. E. Physical activity and overweight among adolescents on the Texas-Mexico Border. Rev. Panam. Salud Publica, 19(4):24452, 2006.

Perichart-Perera, O.; Balas-Nakash, M.; Ortiz-Rodríguez, V.; MoránZenteno, J.A.; Guerrero-Ortiz, J. L. \& Vadillo-Ortega, F. A program to improve some cardiovascular risk factors in Mexican school age children. Salud Publica Mex., 50(3):218-26, 2008.
Ogden, C. L.; Carroll, M. D.; Curtin, L. R.; McDowell, M. A.; Tabak, C. J. \& Flegal, K. M. Prevalence of Overweight and Obesity in the United States, 1999-2004. JAMA, 295(13):1549-55, 2006.

Ogden, C. L.; Flegal, M. K.; Carrol, M. D. \& Jonson, C. L. Prevalence and trends in overweight among US children and adolescents, 19992000. JAMA, 288(14):1728-32, 2002.

Olaiz, G.; Rivera, J.; Shamah, T.; Rojas, R.; Villapando, S.; Hernández, M. \& Sepúlveda, J. Encuesta Nacional de Salud y Nutrición 2006. Cuernavaca, Instituto Nacional de Salud Pública, 2006.

Ramírez-López, E.; Grijalva-Haro, M.; Valencia, M.; Antonio Ponce, J $\&$ Artalejo, E. Impacto de un programa de desayunos escolares en la prevalencia de obesidad y factores de riesgo cardiovascular en niños sonorenses. Salud Publica Mex., 47(2):126-33, 2005.

Rivera, J. A.; Barquera, S.; Campirano, F.; Campos, I.; Safdie, M. \& Tovar, V. Epidemiological and nutritional transition in Mexico: rapid increase of non-communicable chronic diseases and obesity. Public Health Nutr., 5(1A):113-22, 2002.

Rivera, J.; Cuevas, L.; Shamah, T.; Villalpando, S.; Avila, M. \& Jiménez, A. Encuesta Nacional de Salud y Nutrición 2006. Estado nutricio. Cuernavaca, México, Instituto Nacional de Salud Pública, 2006. pp.83-104.

Sallis, J. F. \& Glanz, K. Physical activity and food environments: solutions to the obesity epidemic. Milbank Q., 87(1):123-54, 2009.

Slaughter, M. H.; Lohman, T. G.; Boileau, R. A.; Horswill, C. A.; Stillman, R. J.; Van Loan, M. D. \& Bemben, D. A. Skinfold equations for estimation of body fatness in children and youth. Hum. Biol., 60(5):709-23, 1988

Story, M.; Nanney, M. S. \& Schwartz, M. B. Schools and obesity prevention: creating school environments and policies to promotehealthy eating and physical activity. Milbank $Q$., 87(1):71100, 2009.

Troiano, R. \& Flegal, K. M. Overweight Children and Adolescents: Description, Epidemiology, and Demographics. Pediatrics, 101(3 Pt. 2):497-504, 1988

Villanueva, S. J. \& Ramírez, M. E. Factores asociados al sobrepeso en estudiantes de 8 a 18 años de áreas suburbanas, Hidalgo, México. Respyn, 5(3), 2005. Disponible en: http://www.respyn.uanl.mx/v/ 3/articulos/factores.htm

Dirección para Correspondencia:

Dra. Paulina Yesica Ochoa Martínez

Escuela de Deportes

Universidad Autónoma de Baja California (UABC)

MÉXICO

Email: pochoal@uabc.edu.mx

Recibido: 29-11-2012

Aceptado: 12-09-2013 